\title{
ESCALAS PANAS DE AFECTO POSITIVO Y NEGATIVO PARA NIÑOS Y ADOLESCENTES (PANASN)
}

\author{
BONIFACIO SANDIN \\ Universidad Nacional de Educación a Distancia, Madrid \\ (Aceptado en diciembre 2002)
}

\begin{abstract}
El PANASN es una versión del Positive and Negative Affect Schedule (PANAS; Watson, Clark y Tellegen (1988) para niños y adolescentes. En el presente estudio presentamos datos sobre la estructura factorial, la fiabilidad y la validez del PANASN basados en una muestra de participantes no clínicos (rango de edad 12-17 años, $N=535$ ). El estudio proporciona apoyo emṕrico a favor de la estructura bidimensional de la prueba (i.e., afecto positivo y negativo), así como también, sobre la fiabilidad, y la validez convergente y discriminante con medidas de autoinforme sobre ansiedad y depresión. Se discute la posible relevancia de esta prueba para diferenciar la ansiedad de la depresión en niños y adolescentes.
\end{abstract}

Palabras clave: PANASN, afecto positivo, afecto negativo, ansiedad, depresión.

\section{The PANAS scales of positive and negative affect for children and adolescents} (PANASN)

The PANASN (Sandin, 1997) is a version of the Positive and Negative Affect Schedule (PANAS; Watson, Clark y Tellegen, 1988) for use with children an adolescents. In this study we present data concerning factor structure, reliability and validity of the PANASN in a sample of nonclinical participants (aged $12-17$ years; $N=535$ ). Support is provided for the two-dimensional structure of the measure (i.e., positive and negative affect), as well as, for its reliabilily, and convergent and discriminant validity with existing self-report measures of childhood anxiety and depression. The possible relevance of this instrument to differentiate anxiety from depression in youngsters is discussed.

Key words: PANASN, positive affect, negative affect, anxiety, depression.

\section{INTRODUCCIÓN}

Recientemente, la diferenciación entre el afecto positivo (AP) y el afecto negativo (AN) ha sido considerada como uno de los aspectos más importantes para la separación conceptual entre la ansiedad y la depresión, tanto para la población adulta (Tellegen, 1985; Sandín et al.,

Correspondencia: Bonifacio Sandin, Facultad de Psicología, Universidad Nacional de Educación a Distancia, c/ Juan del Rosal 10 (Ciudad Universitaria), 28040 Madrid. Correo-e: bsandin@psi.uned.es

Agradecimientos: Desearía expresar mi agradecimiento a Aurora Tavar por su participación en la recogida de la información.
1999), como para la población infantojuvenil (Joiner et al., 1996a; Sandín, 1997). Como originalmente sugirió el primero de estos autores (Tellegen, 1985), si bien tanto la ansiedad como la depresión comparten un elevado AN, únicamente la depresión se caracteriza por asociarse a niveles bajos de AP. Del mismo modo, mientras que los síntomas de ansiedad y depresión, o los diagnósticos de un trastorno de ansiedad o depresivo, se relacionan positivamente con el AN, sólo los síntomas depresivos, o el diagnóstico de depresión, se relacionan (negativamente) con el AP. Posteriormente, basándose en estas ideas, Clark y Watson (1991) esta- 
blecieron el denominado "modelo tripartito" sobre la ansiedad y la depresión.

De acuerdo con el modelo tripartito de la ansiedad y la depresión, los tres componentes del modelo, esto es, el distress general (i.e., el alto AN), la hiperactivación fisiológica y la anhedonia (i.e., el bajo AP), constituyen un marco de referencia adecuado para explicar las diferencias entre los síntomas y/o síndromes de ansiedad y depresión (véase Joiner et al., 1996a; Joiner, Catanzaro y Laurent, 1996b). Tal conceptuación tripartita constituye una importante contribución para el conocimiento de la psicopatología de estos dos importantes conceptos (i.e., ansiedad y depresión). En el modelo tripartito, la ansiedad se caracteriza por elevados niveles de activación fisiológica, mientras que el componente distintivo de la depresión es el bajo AP (bajos niveles de interés, placer, etc.). El afecto negativo, en cambio, constituye el aspecto común a la ansiedad y la depresión, lo cual explica la aparente confusión entre ambos constructos ( $p, e j$. , que las correlaciones entre ansiedad y depresión sean elevadas, o que existan síntomas comunes).

La prueba psicométrica de autoinforme conocida como Positive and Negative Affect Schedule (PANAS) es un instrumento breve (consta de 20 items) desarrollado por Watson, Clark y Tellegen (1988) para evaluar el AP y el AN. La idea central para la construcción del PANAS fue la obtención de una medida del afecto basada en descriptores "puros», esto es, basada en marcadores bien del afecto positivo o bien del afecto negativo. Los autores aislaron 10 marcadores para cada una de estas dos dimensiones de la afectividad, los cuales constituyeron los 20 items totales de que consta el PANAS. El PANAS ha sido traducido a muchos idiomas, incluido el español. La adaptación española del PANAS ha sido llevada a cabo conjuntamente por los grupos de
Sandín (Sandín et al., 1999) y Joiner (Joiner et al., 1997, 1998).

El PANAS, a pesar de ser un instrumento de gran interés y de enorme aceptación internacional, es una prueba diseñada para ser aplicada a personas adultas, lo que sugiere que no es adecuada para niños y adolescentes. Sin embargo, la diferenciación entre la ansiedad y la depresión en este tipo de población en una cuestión de especial interés y relevancia psicopatológica y en general clínica (véase, p,ej., Joiner et al., 1996a), ya que la distinción entre ambos conceptos es aun más complicada que en el adulto (p.ej., Brady y Kendall, 1992). Parecía necesario, por tanto, la construcción de una escala similar a la PANAS pero diseñada para ser aplicada a niños y adolescentes.

El primer cuestionario diseñado de forma específica con esta finalidad fue el PANAS para niños y adolescentes (PANASN; véase el Anexo I), el cual fue construido por Sandín (1997) a partir de la versión para adultos de Watson et al. (1988). Datos preliminares sobre la estructura factorial de esta prueba fueron presentados por Romero, Sandín y Chorot (1997). El PANASN es similar en su estructura y contenido al PANAS, ya que incluye los mismos items (20 elementos; 10 sobre AP y 10 sobre AN) que este último. No obstante, la redacción de los elementos del PANASN se llevó a cabo de forma que, sin perder el significado original, pudieran ser comprendidos por los niños y adolescentes. Posteriormente, Laurent et al. (1999) publicaron un cuestionario similar al PANASN aunque algo más largo (30 elementos).

Mediante el presente estudio se pretende proporcionar información empírica sobre la estructura factorial, la fiabilidad y la validez del PANASN, partiendo de una población no clínica. De acuerdo con nuestras predicciones, la estructura factorial debería consistir 
en las dos dimensiones básicas del afecto, esto es, el afecto positivo y el afecto negativo. Estos factores deberían ser independientes, por lo que la correlación entre ambos debería ser baja o despreciable. Los análisis correlacionales entre las escalas del PANASN y determinadas variables de ansiedad y depresión deberían indicar la existencia de correlaciones positivas entre estas últimas variables y el AN, y correlaciones negativas entre dichas variables y el AP. Así mismo, de acuerdo con el modelo tripartito del afecto, la ansiedad y la depresión, el afecto positivo debería correlacionar (negativamente) de forma más elevada con la depresión que con las variables de ansiedad.

\section{MÉTODO}

\section{Participantes}

En la investigación participaron 535 niños y adolescentes normales (no clínicos ni con retraso mental). La distribución por sexo fue de 202 chicos y 333 chicas, con edades comprendidas entre los 7 y los 17 años $(M=14,4, D T=1,7)$. Entre los participantes predominaban los niveles socioeconómicos medio o mediobajo, y asistían regularmente a colegios públicos de enseñanza primaria o secundaria ubicados en la Comunidad de Navarra.

\section{Pruebas psicológicas y procedimiento}

Las pruebas fueron aplicadas a los niños en sus propios centros docentes, a los cuales asistían con regularidad durante el curso académico. Dicha aplicación se llevó a cabo por una psicóloga estudiante de doctorado enla Universidad Nacional de Educación a Distancia. Las pruebas aplicadas fueron las siguientes:
Cuestionario PANAS (Positive and Negative Affect Schedule) para Niños y Adolescentes, PANASN (Sandín, 1997). Prueba elaborada por Sandín (1997) para niños y adolescentes a partir de la forma para adultos del PANAS de Watson, Clark y Tellegen (1988). El PANASN, al igual que el PANAS, es un cuestionario de autoinforme de 20 elementos. Diez items evalúan el afecto positivo (p.ej., "Soy una persona animada, suelo emocionarme") y otros diez el afecto negativo (p.ej., "Me siento nervioso»). El cuestionario es cumplimentado por el niño/adolescente teniendo en cuenta la manera en que éste se siente y/o comporta habitualmente, siguiendo una escala de tres alternativas de respuesta, descritas éstas como "Nunca» (1), «A veces» (2), y «Muchas veces» (3). (Véase el Anexo I).

Indice de Sensibilidad a la Ansiedad para Niños [Childhood Anxiety Sensitivity Index] (CASI, Silverman, Fleisig, Rabian y Peterson, 1991). Se aplicó la versión española de Sandín y Chorot (Sandín, 1997). Consta de 18 items que se puntúan según una escala Likert de 3 puntos: Nada (1), Un poco (2), Mucho (3). La puntuación total se obtiene sumando las puntuaciones obtenidas en los 18 items. Los items se refieren a consecuencias físicas (p.ej., «Me asusto cuando mi corazón late rápidamente»), cognitivas (p.ej., "Me asusto cuando no puedo concentrarme en los deberes de clasen), y sociales (p.ej., "Me gusta no mostrar mis sentimientos a los demás ). Datos sobre la fiabilidad, validez y estructura factorial de la versión española de la CASI han sido obtenidos por Sandín, Chorot, Santed y Valiente (2002), y Sandín, Chorot, Valiente y Santed (2002).

Cuestionario de Ansiedad Estado/Rasgo para Niños STAIC [State-Trait Anxiety Inventory for Children] (STAIC, Spielberger, 1970). Se aplicó la versión española adaptada por TEA (Spielberger, 1988). 
Utilizamos la Parte 2, la cual evalúa específicamente el rasgo de ansiedad (p.ej., «Me cuesta enfrentarme a mis problemas», "Me preocupan las cosas del colegio",). Dicha parte consta de 20 items que son contestados por los participantes indicando cómo se sienten habitualmente, según una escala de tres niveles: «Casi nunca» (1), "A veces» (2) y «A menudo» (3).

Cuestionario de Depresión para Niños, $C D N$ (Sandín y Valiente, 1996). El CDN es una prueba de autoinforme elaborada por Sandín y Valiente (1996) con objeto de obtener una evaluación fácil y breve sobre la depresión que experimentan los niños y adolescentes. Más que dirigido a medir el estado de ánimo depresivo, el CDN fue diseñado para evaluar el nivel de depresión clínica. Consta de 16 items, los cuales se refieren a las principales facetas de los síntomas depresivos (anhedonia, ánimo depresivo, desesperanza, inhibición cognitiva, y manifestaciones físicas). La cumplimentación del cuestionario se lleva a cabo teniendo en cuenta la frecuencia de sentimientos, pensamientos o conductas que le hayan ocurrido al sujeto durante las últimas semanas, debiendo señalar, para cada elemento, una entre las tres alternativas de respuesta siguientes: «Nunca» (1), «A veces" (2), o "Muchas veces" (3). Se obtiene una puntuación global en depresión sumando la puntuación obtenida en cada ítem. Se han informado coeficientes alfa para el CDN entre 0,77 y 0,78 (Valiente, Sandín y Chorot, 2002; Valiente, Sandín, Chorot y Tabar, 2002).

\section{RESULTADOS}

\section{Análisis factorial del PANASN}

El análisis factorial se llevó a cabo con la muestra total sobre los 20 elementos del PANASN siguiendo el método de análi- sis de componentes principales. Se efectuó inicialmente la rotación ortogonal (varimax), ya que la evidencia de la literatura con la forma para adultos ha sugerido consistentemente una estructura de dos factores bastante independientes. No obstante se llevó a cabo también la rotación oblicua (oblimin) para obtener la correlación entre los factores y contrastar posibles cambios en la estructura con la solución oblicua. Para la extracción del número de factores partimos en primer lugar de nuestra asunción de 2 factores como base de la estructura simple del PANASN. También aplicamos los siguientes criterios: (a) el test de scree (Cattell, 1966), (b) los criterios de extracción de Thurstone (1947), y (c) los criterios de interpretabilidad de la estructura simple de Gorsuch (1983). El índice de Kaiser-Meyer-Olkin $(=0,812)$ sugería que los datos eran adecuados para el cálculo del análisis factorial.

En general todos los criterios, tanto los criterios empíricos como el criterio teórico, sugerían que la mejor solución factorial consistía en una estructura de dos factores. Dichos factores representaban bastante bien las dos escalas del cuestionario, esto es, las dimensiones del afecto positivo y negativo. Aunque se obtuvieron las soluciones factoriales correspondientes a tres y cuatro factores, éstas no resultaban adecuadas al aplicar los criterios de extracción, por lo que fueron desechadas como posibles alternativas a la estructura de dos factores.

En la Tabla 1 presentamos los resultados correspondientes al análisis factorial de dos factores tras la rotación varimax. Los factores, que explicaban el $32,5 \%$ de la varianza total, representan de forma muy coherente la estructura bidimensional del PANASN. Como puede observarse, todos los items obtienen saturaciones relativamente elevadas en el factor relevante (la mayoría están por encima del valor de $\mathbf{0 , 4 0}$ ). Por otra parte, no se ha 
Tabla 1. Análisis de componentes principales del PANASN tras rotación ortogonal (varimax). Pesos factoriales de las dos dimensiones aisladas, correspondientes al AP y $\mathrm{AN}(N=535)$

\begin{tabular}{|c|c|c|c|}
\hline Elemento del PANASN & $\begin{array}{c}\text { AN } \\
\text { Factor } 1 \\
\end{array}$ & $\begin{array}{c}\text { AP } \\
\text { Factor } 2 \\
\end{array}$ & $\begin{array}{c}\text { Correlación } \\
\text { [tem-total }^{1} \\
\end{array}$ \\
\hline 1. Me intereso por la gente o las cosas & 0,24 & $\mathbf{0 , 4 1}$ & 0,26 \\
\hline 2. Me siento tenso/a, agobiado/a, con sensación de estrés & 0,58 & $-0,11$ & 0,44 \\
\hline 3. Soy una persona animada, suelo emocionarme & $-0,10$ & 0,64 & 0,45 \\
\hline 4. Me siento disgustado/a o molesto/a & $\mathbf{0 , 5 4}$ & $-0,20$ & 0,44 \\
\hline 5. Siento que tengo vitalidad o energía & 0,19 & 0,69 & 0,53 \\
\hline 6. Me siento culpable & $\mathbf{0 , 6 0}$ & 0,01 & 0,46 \\
\hline 7. Soy un/a chico/a asustadizo/a & $\mathbf{0 , 5 2}$ & $-0,08$ & 0,41 \\
\hline 8. Estoy enfadado/a o furioso/a & $\mathbf{0 , 4 3}$ & $-0,14$ & 0,37 \\
\hline 9. Me entusiasmo (por cosas, personas, etc.) & 0,29 & $\mathbf{0 , 4 3}$ & 0,25 \\
\hline 10. Me siento orgulloso/a (de algo), satisfecho/a & $-0,21$ & $\mathbf{0 , 6 0}$ & 0,45 \\
\hline 11. Tengo mal humor (me altero o irrito) & $\mathbf{0 , 5 0}$ & $-0,16$ & 0,40 \\
\hline 12. Soy un/a chico/a despierto/a, "despabilado/a» & $-0,21$ & $\mathbf{0 , 5 1}$ & 0,39 \\
\hline 13. Soy vergonzoso/a & 0,45 & $-0,15$ & 0,35 \\
\hline 14. Me siento inspirado/a & $-0,12$ & $\mathbf{0 , 4 1}$ & 0,32 \\
\hline 15. Me siento nervioso/a & $\mathbf{0 , 5 9}$ & 0,05 & 0,46 \\
\hline 16. Soy un/a chico/a decidido/a & $-0,30$ & $\mathbf{0 , 5 1}$ & 0,40 \\
\hline 17. Soy una persona atenta, esmerada & 0,25 & $\mathbf{0 , 3 1}$ & 0,19 \\
\hline \multicolumn{4}{|l|}{ 18. Siento sensaciones corporales de estar intranquilo/a o } \\
\hline preocupado/a & 0,69 & 0,02 & 0,54 \\
\hline 19. Soy un/a chico/a activo/a & $-0,13$ & $\mathbf{0 , 6 6}$ & 0,50 \\
\hline 20. Siento miedo & 0,60 & $-0,07$ & 0,49 \\
\hline Autovalores & 4,13 & 2,34 & \\
\hline$\%$ de varianza explicada & 20,70 & 11,80 & \\
\hline
\end{tabular}

Nota. ' La correlación ítem-total se refiere a la correlación corregida entre el f́tem en cuestión y la escala de AP o AN (las saturaciones en negrita indican los items que constituyen cada escala). AP = afecto positivo, AN = afecto negativo.

producido ninguna saturación de 0,30 o superior en el factor no relevante (es decir, elementos de AP que saturen en $A N$, y elementos de AN que saturen en AP). Estos datos, junto a la evidencia obtenida a partir de las correlaciones (corregidas) entre cada ítem y la escala relevante (i.e., correlaciones ítem-total), indican que, en general, la PANASN posee una clara estructura bidimensional que se corresponde con la estructura básica del afecto y confiere validez a la estructura interna del cuestionario. La correlación entre los factores fue de $-0,13$, es decir, en la línea de lo esperado, y similar a las obtenidas en nuestros anteriores estudios con la versión para adultos (p.ej., Sandín et al., 1999; en este estudio, las correlaciones entre ambos factores oscilaron entre 0,08 y $-0,17$ ).

Puede observarse, no obstante, que algunos items parecen contribuir mejor que otros a cada una de las dimensiones. Si tomamos como punto de referencia los valores de las saturaciones y de las correlaciones ítem-total (Tabla 1), parece evidenciarse que, en general, la dimensión del AN parece más consistente que la del AP. Las variables que más parecen contribuir al factor de AN son las correspondientes a los Items 18 (intranquilidad), 20 (temor), 6 (culpabilidad), 15 (nerviosismo) y 2 (tensión); las que menos, corresponden a los Items 8 (enfado) y 13 (vergüenza). Las variables que más contribuyen al factor de AP son las que corresponden a los Items 5 (energía), 19 (actividad), y 3 (ánimo); las menos consistentes con el factor corresponden a los items 17 (atención), 9 (entusiasmo) y 1 (interés). No obstante, aun así, la estructura general es bastante consistente $y$ muy coherente con el modelo teórico del afecto en el que se apoya el cuestionario. 
Tabla 2. Diferencias sexuales y coeficientes alfa de Cronbach para AP y AN (entre paréntesis se indican las desviaciones típicas)

\begin{tabular}{lccc}
\hline & Chicos & \multicolumn{2}{c}{ Chicas } \\
\cline { 2 - 4 } Variable (rango de puntuaciones) & Media (DT) & Media $(D T)$ & $t(533)$ \\
\hline Afecto positivo (AP) (10-30) & $24,3(3,07)$ & $24,2(2,97)$ & 1,76 \\
Afecto negativo (AN) (10-30) & $19,1(3,17)$ & $21,2(3,23)$ & $-7,22^{\star \star \star}$ \\
\hline
\end{tabular}

Nota. ${ }^{\star \star \star} p<0,001$.

Esto vendría a indicar que algunos elementos podrían actuar más claramente como auténticos "marcadores» del afecto positivo o negativo, mientras que otros podrían tener un menor peso o significación. En nuestro anterior estudio con estudiantes universitarios (Sandín et al., 1999), vimos que los más destacados marcadores del afecto negativo fueron la intranquilidad, el temor y el nerviosismo, mientras que los principales marcadores del afecto positivo fueron los elementos relacionados con energía y actividad; como puede apreciarse, los "marcadores de factor" prácticamente coinciden en ambos estudios.

\section{Medias y desviaciones típicas}

En la tabla 2 se indican las medias y desviaciones típicas correspondientes al AP y AN en función del sexo. Como puede apreciarse, no existen diferencias entre chicos y chicas en AP, pero sí en AN ( $p<$ 0,001 ). Así mismo, las puntuaciones tienden a ser más elevadas en afecto positivo que en afecto negativo. Estos resultados corroboran nuestros anteriores hallazgos con muestras de adultos basados en la versión española del PANAS (Joiner et al., 1997; Sandín et al., 1999).

Fiabilidad (alfa) y validez convergente y discriminante del PANASN

En la Tabla 3 indicamos los coeficientes alfa de Cronbach y las correlaciones entre las escalas de la afectividad y otras variables de ansiedad y depresión. Tal y como reflejamos en dicha tabla, los coeficientes de fiabilidad (consistencia interna) de Cronbach son aceptables; no alcanzan el valor de $\mathbf{0 , 8 0}$, pero todos los coeficientes están por encima de 0,70. Con la versión española del PANAS se han obtenido coeficientes más elevados (todos superiores a 0,80; Sandín et al., 1999).

Las correlaciones entre las dimensiones del PANASN y la ansiedad y la depresión constatan que, mientras que el afecto negativo se relaciona de forma positiva y bastante elevada con la ansiedad, la depresión y la sensibilidad a la ansiedad, el afecto negativo se relaciona de forma negativa con estas variables. Este patrón general de correlaciones es similar en ambos grupos (chicos y chicas). Como se indica en la Tabla 3, las correlaciones más elevadas del AN se producen con el rasgo de ansiedad y, en el grupo de chicas, con la depresión. Las correlación más elevada (negativa) del AP ocurre con la depresión (en ambos grupos).

Estas correlaciones proporcionan evidencia preliminar en apoyo de la validez convergente $y$ discriminante del PANASN, ya que la variable AN correlaciona de forma positiva con otras variables de emocionalidad negativa (ansiedad, depresión y sensibilidad a la ansiedad) (validez convergente), mientras que el AP correlaciona negativamente con la ansiedad, la depresión y la sensibilidad a la ansiedad (validez discriminante) (en este sentido, llama particular- 
Tabla 3. Correlaciones de Pearson entre las variables del PANASN (AP y AN) y las variables de ansiedad y depresión, y coeficientes alfa de Cronbach

\begin{tabular}{lcccc}
\hline & \multicolumn{2}{c}{ Chicos $(N=2002)$} & \multicolumn{2}{c}{ Chicas $(N=333)$} \\
\cline { 2 - 5 } & AP & AN & AP & AN \\
\hline Sensibilidad a la ansiedad (CASI) & $-0,15^{\star}$ & $0,45^{\star \star}$ & $-0,14^{\star}$ & $0,44^{\star \star}$ \\
Rago de ansiedad (STAIC) & $-0,22^{\star \star}$ & $0,60^{\star \star}$ & $-0,29^{\star \star}$ & $0,67^{\star \star}$ \\
Depresión (CDN) & $-0,43^{\star \star}$ & $0,45^{\star \star}$ & $-0,39^{\star \star}$ & $0,57^{\star *}$ \\
Coeficiente alfa de Cronbach & 0,73 & 0,74 & 0,72 & 0,75 \\
\hline
\end{tabular}

Nota. ${ }^{\star} p<0,05 ;{ }^{\star \star} p<0,01 ; \mathrm{AP}=$ afecto positivo, $\mathrm{AN}=$ afecto negativo

mente la atención que el AN correlacione especialmente con depresión).

Las correlaciones entre las escalas de AP y AN fueron de -0,14 (grupo de chicos) y -0,33 (grupo de chicas). En principio, esta última correlación observada para el grupo de chicas resulta algo elevada si tenemos en cuenta que partimos de que se trata de dos dimensiones independientes (la correlación del grupo de chicos parece, en cambio, bastante coherente) (aunque se postula que se trata de dos dimensiones ortogonales, la mayor parte de la evidencia de la literatura indica que el AP y el AN tienden a correlacionar ligeramente entre sí de forma negativa).

\section{DISCUSIÓN}

En el presente estudio se ha analizado la estructura factorial del PANASN, así como su fiabilidad y validez. También se ha pretendido obtener información sobre estadísticos descriptivos (medias y desviaciones típicas), comparando en función de los grupos de sexo. Nuestras hipótesis central sugería que el PANASN debería tener una estructura bifactorial coherente con las dimensiones (escalas) de AP y AN, debiendo ser estas dimensiones relativamente independientes. Además, las correlaciones de estas dos escalas con otras variables de ansiedad y depresión deberían servir para contrastar la validez convergente y divergente.
La evidencia basada en el análisis factorial constata la validez de la estructura del cuestionario, la cual es consistente con la abundante literatura sobre el PANAS que demuestra la supremacía de dos factores del afecto relativamente independientes (p. ej., Tellegen, 1985; Watson et al., 1988; Joiner et al., 1996a; Sandín et al., 1999). Los datos del presente estudio ponen de relieve que la estructura del afecto en niños y adolescentes es similar a la del adulto, por lo que la PANASN podría utilizarse para evaluar el afecto de forma paralela al empleo del PANAS con personas adultas. Así mismo, los resultados confirman nuestros datos preliminares sobre la estructura del PANASN (Romero et al., 1997), y son consistentes con la evidencia obtenida por Laurent et al. (1999) partiendo de un cuestionario similar.

Aunque en general los datos indican que la estructura factorial es bastante coherente, tal y como ha ocurrido con otros estudios, sin embargo no todos los elementos del cuestionario parecen tener la misma significación o peso (algunos elementos parecen representar mejor que otros el afecto positivo o negativo). Ya indicamos atrás que ciertos elementos del cuestionario parecen contribuir de forma mucho más específica en la configuración del factor (AP o AN), lo cual sintoniza con nuestros anteriores datos obtenidos a partir de estudiantes universitarios (Sandín et al., 1999). Aparte del propio interés conceptual que conlleva, esto podría 
ser importante si se desease emplear una forma abreviada de la prueba.

Los datos sobre la fiabilidad y la validez indican que la prueba posee aceptables propiedades, tanto en lo que concierne a la fiabilidad (consistencia interna) como a la validez convergente y divergente. Es indudable que se necesitan aún nuevos estudios para probar otras formas de fiabilidad (p. ej., testretest) y validez (validez discriminante respecto a síntomas y/o trastornos de ansiedad y depresión) del cuestionario. En lo que concierne a las diferencias de medias, donde sólo se constatan diferencias significativas para el afecto negativo, nuestros resultados vienen a confirmar la ya conocida asociación entre autoinforme de emocionalidad negativa y sexo femenino.

En relación con la información que hemos obtenido sobre la validez discriminante de la aprueba, nos gustaría subrayar el hecho de que el AP haya correlacionado de forma bastante más elevada con depresión que con cualquiera de las dos variables de ansiedad (rasgo de ansiedad y sensibilidad a la ansiedad). Este resultado es importante, ya que proporciona apoyo parcial al modelo tripartito sobre el afecto, la ansiedad y la depresión (Clark y Watson, 1991), ya que, según este modelo, mientras que el afecto negativo se relaciona de forma similar con la ansiedad y la depresión, el bajo afecto positivo es más característico de la depresión (i.e., como se demuestra en nuestro trabajo por unas correlaciones negativas más elevadas entre AP y depresión que entre AP y ansiedad).

En suma, el PANASN es el primer cuestionario construido específicamente para la evaluación de la afectividad en niños y adolescentes basado en el modelo bidimensional del afecto (i.e., afecto positivo versus negativo) (Tellegen, 1985; Watson et al., 1988). El cuestionario constituye una forma paralela, para ser aplicada en niños y adolescentes, del cuestionario para adultos PANAS. Dada la relevancia internacional que ha tenido este último cuestionario, pensamos que el PANASN puede resultar de enorme interés para el estudio de la ansiedad y la depresión en niños y adolescentes, y especialmente para la diferenciación entre estos dos constructos psicológicos desde el marco del modelo tripartito (p.ej., Joiner et al., 1996a, 1996b). Por otra parte, el cuestionario siempre podrá ser utilizado para obtener una evaluación de la afectividad en el niño, con independencia de que se investigue o no la ansiedad y la depresión.

\section{REFERENCIAS BIBLIOGRÁFICAS}

Brady, E.U., y Kendall, P.C. (1992). Comorbidity of anxiety and depression in children and adolescents. Psychological Bulletin, 111, 244-255.

Cattell, R.B. (1966). The scree test for the number of factors. Multivariate Behavioral Research, 3, 245-276.

Clark, L.A., y Watson, D. (1991). Tripartite model of anxiety and depression: Psychometric evidence and taxonomic implications. Journal of Abnormal Psychology, 100, 316-336.

Gorsuch, R. (1983). Factor analysis. Hillsdale, NJ: Erlbaum.

Joiner, T.E., Catanzaro, S.J., y Laurent, J. (1996b). Tripartite structure of positive and negative affect, depresión, and anxiety in child and adolescent psychatric inpatients. Journal of Abnormal Psychology, 105, 401409.

Joiner, T.E., Catanzaro, S.J., Laurent, J., Sandín, B., y Blalock, J.A. (1996a). Modelo tripartito sobre el afecto positivo y negativo, la depresión y la ansiedad: Evidencia basada en la estructura de los síntomas y en diferencias sexuales. Revista de Psicopatologia y Psicología Clínica, 1, 27-34.

Joiner, T.E., Sandín, B., Chorot, P., Lostao, L., y Marquina, G. (1997). Development and factor-analytic validation of the SPANAS 
among women in Spain: (More) cross-cultural convergence in the structure of mood. Journal of Personality Assessment, 68, 600615.

Joiner, T.E., Sandín, B., Chorot, P., Lostao, L., Santed, M.A., Catanzaro, S.J., Laurent, y J., Marquina, G. (1998). A confirmatory factor-analytic validation of the tripartite model of depression and anxiety among undergraduates in Spain. Journal of Gender, Culture and Health, 3, 147-157.

Laurent, J., Catanzaro, S., Joiner, T.E., Rudolph, K.D., Potter, K.I., Lambert, S., Osborne, L., y Gathright, T. (1999). A measure of positive and negative affect for children: Scale development and preliminary validation. Psychological Assessment, 11, 326-338.326-338.

Romero, M., Sandín, B., y Chorot, P. (1997). Datos preliminares con el cuestionario de afecto positivo y negativo para niños y adolescentes (PANASN). Presentado al I Congreso de la Asociación Española de Psicología Clínica y Psicopatología. Madrid, 3-5 de abril.

Sandín, B., Chorot, P., Lostao, L., Joiner, T.E., Santed, M.A., y Valiente, R.M. (1999). Escalas PANAS de afecto positivo y negativo: Validación factorial y convergencia transcultural. Psicothema, 11, 37-51.

Sandín, B., Chorot, P., Santed, M.A., y Valiente, R.M. (2002). Análisis factorial confirmatorio del f́ndice de Sensibilidad a la Ansiedad para Niños. Psicothema, 14, 333339.

Sandín, B., Chorot, P., Valiente, R.M., y Santed, M.A. (2002). Relación entre la sensibi- lidad a la ansiedad y el nivel de miedos en niños. Psicología Conductual, 10, 107-120. Sandín, B., y Valiente, R.M. (1996). Cuestionario de Depresión en Niños. Madrid: Universidad Nacional de Educación a Distancia (policopiado).

Silverman, W.K., Fleisig, W., Rabian, B., y Peterson, R.A. (1991). Childhood Anxiety Sensitivity Index. Journal of Clinical Child Psychology, 20, 162-168.

Spielberger, C.D. (1988). Cuestionario de Ansiedad Estado/Rasgo en Niños (STAIC). Madrid: TEA (orig. inglés 1970).

Tellegen, A. (1985). Structures of mood and personality and their relevance to assessing anxiety, with emphasis on self-report. En A.H. Tuma y D. Maser (Eds.), Anxiety and the anxiety disorders (pp. 681-706). Hillsdale, NJ: Erlbaum.

Thurstone, L.L. (1947). Multiple factor analysis. Chicago: University of Chicago Press.

Valiente, R.M., Sandín, B., y Chorot, P. (2002a). Miedos comunes en niños y adolescentes: Relación con la sensibilidad a la ansiedad, el rasgo de ansiedad, la afectividad negativa y la depresión. Revista de Psicopatología y Psicología Clínica, 7, 61-70.

Valiente, R.M., Sandín, B., y Chorot, P. (2002b). Miedos comunes en niños y adolescentes: Su relación con la sensibilidad a la ansiedad y otras emociones negativas. Psiquis, 23, 217-225.

Watson, D., Clark, L.A., y Tellegen, A. (1988). Development and validation of brief measures of positive and negative affect: The PANAS scales. Journal of Personality and Social Psychology, 54, 1063-1070. 


\section{ANEXO I. CUESTIONARIO PANASN}

Cuestionario PANAS para niños y adolescentes (PANASN)

B. Sandín, 1997

NOMBRE

.EDAD

SEXO:

$\square$ Chico

Chica

Instrucciones: A continuación se indican algunas frases que los chicos y chicas utilizan para describirse a sí mismos. Lee detenidamente cada frase y marca cada una de ellas con una $\alpha \mathbf{X}$ en el espacio correspondiente a una de las tres alternativas (Nunca, A veces o Muchas veces). No existen contestaciones buenas ni malas. Recuerda que tienes que señalar la alternativa que mejor se ajuste a tu forma de ser.

NUNCA: si nunca o casi nunca sientes o te comportas de la manera que dice la frase

A VECES: si en algunas ocasiones sientes o te comportas como indica la frase MUCHAS VECES: si la mayor parte del tiempo sientes o te comportas como dice la frase

\begin{tabular}{|c|c|c|c|c|}
\hline 1. & Me intereso por la gente o las cosas & $\square$ NUNCA & $\square$ A VECES & 口 MUCHAS VECES \\
\hline 2. & Me siento tenso/a, agobiado/a, con sensación de estrés & $\square$ NUNCA & $\square$ A VECES & 口 MUCHAS VECES \\
\hline 3. & Soy una persona animada, suelo emocionarme & 口 NUNCA & $\square$ A VECES & 口 MUCHAS VECES \\
\hline 4. & Me siento disgustado/a o molesto/a & $\square$ NUNCA & $\square$ A VECES & $\square$ MUCHAS VECES \\
\hline 5. & Siento que tengo vitalidad o energía & $\square$ NUNCA & $\square$ A VECES & 口 MUCHAS VECES \\
\hline 6. & Me siento culpable & $\square$ NUNCA & $\square$ A VECES & $\square$ MUCHAS VECES \\
\hline 7. & Soy un/a chico/a asustadizo/a & $\square$ NUNCA & $\square$ A VECES & D MUCHAS VECES \\
\hline 8. & Estoy enfadado/a o furioso/a & $\square$ NUNCA & $\square$ A VECES & 口 MUCHAS VECES \\
\hline 9. & Me entusiasmo (por cosas, personas, etc.) & $\square$ NUNCA & 口 A VECES & $\square$ MUCHAS VECES \\
\hline 10. & Me siento orgulloso/a (de algo), satisfecho/a & D NUNCA & $\square$ A VECES & $\square$ MUCHAS VECES \\
\hline 11. & Tengo mal humor (me altero o irrito) & $\square$ NUNCA & $\square$ A VECES & $\square$ MUCHAS VECES \\
\hline 12. & Soy un/a chico/a despierto/a, "despabilado/a" & $\square$ NUNCA & $\square$ A VECES & $\square$ MUCHAS VECES \\
\hline 13. & Soy vergonzoso/a & 口 NUNCA & 口 A VECES & $\square$ MUCHAS VECES \\
\hline 14. & Me siento inspirado/a & $\square$ NUNCA & 口 A VECES & $\square$ MUCHAS VECES \\
\hline 15. & Me siento nervioso/a & $\square$ NUNCA & $\square$ A VECES & $\square$ MUCHAS VECES \\
\hline 16. & Soy un/a chico/a decidido/a & $\square$ NUNCA & $\square$ A VECES & D MUCHAS VECES \\
\hline 17. & Soy una persona atenta, esmerada & $\square$ NUNCA & D A VECES & D MUCHAS VECES \\
\hline 18. & Siento sensaciones corporales de estar intranquilo/a & & & \\
\hline & o preocupado/a & $\square$ NUNCA & $\square$ A VECES & DUCHAS VECES \\
\hline 19. & Soy un/a chico/a activo/a & $\square$ NUNCA & $\square$ A VECES & D MUCHAS VECES \\
\hline 20. & Siento miedo & $\square$ NUNCA & $\square$ A VECES & $\square$ MUCHAS VECES \\
\hline
\end{tabular}

Subescalas: Afecto positivo (AP, items 1, 3, 5, 9, 10, 12, 14, 16, 17, 19); Afecto negativo (AN, items 2, 4, 6, 7, 8, 11, 13, $15,18,20)$. Tomado de Sandín (1997, p. 19) (reproducido con permiso). 